\title{
Growth and structure of singly oriented single-layer tungsten disulfide on $\mathrm{Au}(111)$
}

\author{
Luca Bignardi, ${ }^{1, *}$ Daniel Lizzit, ${ }^{1}$ Harsh Bana,${ }^{2, \dagger}$ Elisabetta Travaglia, ${ }^{2}$ Paolo Lacovig, ${ }^{1}$ Charlotte E. Sanders, $, 3, \dagger$ \\ Maciej Dendzik, ${ }^{3, \S}$ Matteo Michiardi, ${ }^{3, \|}$ Marco Bianchi, ${ }^{3}$ Moritz Ewert, ${ }^{4,5}$ Lars Buß, ${ }^{4}$ Jens Falta, ${ }^{4,5}$ Jan Ingo Flege, ${ }^{4,5, \mathbb{\Psi}}$ \\ Alessandro Baraldi, ${ }^{1,2,6}$ Rosanna Larciprete, ${ }^{7}$ Philip Hofmann, ${ }^{3, \#}$ and Silvano Lizzit ${ }^{1, * *}$ \\ ${ }^{1}$ Elettra-Sincrotrone Trieste S.C.p.A., AREA Science Park, Strada Statale 14, km 163.5, 34149 Trieste, Italy \\ ${ }^{2}$ Department of Physics, University of Trieste, Via Valerio 2, 34127 Trieste, Italy \\ ${ }^{3}$ Department of Physics and Astronomy, Interdisciplinary Nanoscience Center (iNANO), Aarhus University, \\ Ny Munkegade 120, 8000 Aarhus C, Denmark \\ ${ }^{4}$ Institute of Solid State Physics, University of Bremen, Otto-Hahn-Allee 1, 28359 Bremen, Germany \\ ${ }^{5}$ MAPEX Center for Materials and Processes, Bremen, Germany \\ ${ }^{6}$ IOM-CNR, Laboratorio TASC, AREA Science Park, Strada Statale 14, km 163.5, 34149 Trieste, Italy \\ ${ }^{7}$ CNR-Institute for Complex Systems, Via dei Taurini 19, 00185 Roma, Italy
}

(Received 19 September 2018; published 22 January 2019)

\begin{abstract}
A singly oriented, single layer of tungsten disulfide $\left(\mathrm{WS}_{2}\right)$ was epitaxially grown on $\mathrm{Au}(111)$ and characterized at the nanoscale by combining photoelectron spectroscopy, photoelectron diffraction, and low-energy electron microscopy. Fast x-ray photoelectron spectroscopy revealed that the growth of a single crystalline orientation is triggered by choosing a low $\mathrm{W}$ evaporation rate and performing the process with a high temperature of the substrate. Information about the single orientation of the layer was obtained by acquiring x-ray photoelectron diffraction patterns, revealing a $1 \mathrm{H}$ polytype for the $\mathrm{WS}_{2}$ layer and, moreover, determining the structural parameters and registry with the substrate. The distribution, size, and orientation of the $\mathrm{WS}_{2}$ layer were further ascertained by low-energy electron microscopy.
\end{abstract}

DOI: 10.1103/PhysRevMaterials.3.014003

\section{INTRODUCTION}

Only a short time after the successful creation of graphene by mechanical exfoliation [1-3], it was realized that other two-dimensional materials could be obtained in a similar way from layered crystals, especially transition-metal dichalcogenides (TMDCs) [4]. This has led to an intense study of single-layer (SL) TMDCs, which, like graphene, show electronic properties that are remarkably different from those of their parent bulk compound. Initially, much attention had been given to the presence of a direct band gap in some of the semiconducting SL TMDCs [5,6] and the possibility to exploit

\footnotetext{
*Present address: Department of Physics, University of Trieste, via Valerio 2, 34127 Trieste, Italy; lbignardi@units.it

†Present address: Instituut voor Kern- en Stralingsfysica, Department of Physics and Astronomy, KU Leuven, Celestijnenlaan 200D, B-3001 Leuuven, Belgium.

${ }^{\ddagger}$ Present address: Central Laser Facility STFC Rutherford Appleton Laboratory, Harwell, Didcot OX11 0QX, United Kingdom.

${ }^{\S}$ Present address: Department of Physical Chemistry, Fritz-HaberInstitut of the Max Planck Society, Faradayweg 4-6, Berlin 14915, Germany

"Present address: Quantum Matter Institute, University of British Columbia, 2355 East Mall, Vancouver, Canada BC V6T $1 Z 4$.

IPresent address: Institute of Physics, Brandenburg University of Technology Cottbus-Senftenberg, Konrad-Zuse-Strasse, 03046 Cottbus, Germany.

\#philip@phys.au.dk

***izzit@elettra.eu
}

this property in electronic and optoelectronic applications $[7,8]$. Later, it was suggested that these materials could be used for entirely new concepts in electronics because of their coupled spin [9] and valley [10-13] degrees of freedom [14], something that does not play a role in graphene, where inversion symmetry prevents the lifting of the spin degeneracy.

The technological exploitation of the SL TMDCs will benefit from production by bottom-up growth, rather than exfoliation. Chemical vapor deposition and molecular beam epitaxy appear to be promising approaches to obtain a high degree of orientation and a closed layer [15-22]. An additional requirement for devices that shall make use of the valley degree of freedom, such as a valley filter [23], is to avoid the presence of mirror domains, a common defect in the growth of SL TMDCs [19]. The two mirrored versions of the SL TMDC unit cell have the same reciprocal lattice, with merely the $K$ and $-K$ points of the surface Brillouin zone inverted [24] and the same band structure, apart from an inverted spin polarization for the two mirror domains. The presence of mirrored domains is thus detrimental for both fundamental studies on the coupled spin and valley degrees of freedom and for the use of TMDCs in devices [13,25].

There has been recent progress in the growth of single orientation two-dimensional materials, for example for SL BN on $\operatorname{Ir}(111)$ [26], as well as for $\mathrm{SL} \mathrm{MoS}_{2}$ on hexagonal boron nitride [21] and $\mathrm{Au}(111)$ [24]. Herein, we investigated the growth and structural features of epitaxially grown SL $\mathrm{WS}_{2}$ on $\mathrm{Au}(111)$. We revealed that a bottom-up approach can be employed to obtain $\mathrm{SL} \mathrm{WS}_{2}$ with a single orientation, something that has not been reported so far. This aspect, together 
with the spin-splitting in the valence band, which is one of the largest among TMDCs [27-29], opens up the possibility to investigate and exploit the coupled valley and spin degrees of freedom in devices applications. X-ray photoelectron diffraction (XPD) experiments conclusively proved the presence of a single-crystalline orientation of the $\mathrm{WS}_{2}$ layer. Lowenergy electron microscopy (LEEM) showed more features of the morphology and distribution of the $\mathrm{WS}_{2}$ crystalline domains, finding an average size of a few squared microns. Furthermore, these measurements confirm the presence of a single orientation.

\section{EXPERIMENTAL METHODS}

Growth and characterization experiments were carried out at the SuperESCA beamline [30] of the Elettra Synchrotron radiation facility in Trieste, Italy, except for the LEEM data, which were acquired at the University of Bremen. The $\mathrm{Au}(111)$ substrate was cleaned in ultrahigh vacuum by cycles of $\mathrm{Ar}^{+}$ion sputtering at $2 \mathrm{keV}$ and annealing up to $950 \mathrm{~K}$. The cleanliness of the surface was checked by low-energy electron diffraction (LEED) and X-ray photoelectron spectroscopy (XPS), which did not detect traces of contamination. We have grown the singly oriented $\mathrm{WS}_{2}$ single layer on the $\mathrm{Au}(111)$ surface by depositing $\mathrm{W}$ with a rate of $3.3 \times 10^{-3} \mathrm{ML} / \mathrm{min}$, measured by means of a quartz microbalance-1 ML corresponds to the surface atomic density of $\mathrm{Au}(111)$ - with $\mathrm{H}_{2} \mathrm{~S}$ pressure of $1 \times 10^{-5} \mathrm{mbar}$ and a substrate temperature of $923 \mathrm{~K}$. The growth was followed in real time by fast XPS, acquiring the $\mathrm{W} 4 f$ core level during the entire process, easing the optimization of the optimal growth parameters, also thanks to the combination of a high-flux beamline and a highefficiency electron detector. Different growth parameters lead to the presence of $\mathrm{W}$ clusters and partially sulfided species and to the formation of detrimental mirror domains in the final sample. A full account of these measurements is presented in [31].

High-resolution XPS S $2 p$ and W $4 f$ core-level spectra were measured in situ at room temperature in normal emission on the as-grown $\mathrm{WS}_{2}$ single layer, using photon energies of 260 and $140 \mathrm{eV}$, respectively. The overall energy resolution was below $50 \mathrm{meV}$.

The XPD patterns were obtained by collecting $50 \mathrm{az}-$ imuthal scans for a range of polar emission angles $\theta$, from grazing emission $\left(\theta=70^{\circ}\right)$ to normal emission $\left(\theta=0^{\circ}\right)$. An azimuthal scan consists of the acquisition over a wide azimuthal sector $\left(\phi=130^{\circ}\right)$ in either the $\mathrm{W} 4 f_{7 / 2}$ or $\mathrm{S} 2 p_{3 / 2}$ core-level region. For each of these spectra, a peak fit analysis was performed (parameters of the analysis reported in [31]), and the intensity $I(\theta, \phi)$ of each component resulting from the fit, i.e., the area under the photoemission line, was extracted. The resulting XPD patterns are the stereographic projection of the modulation function $\chi$, which was obtained from the peak intensity for each emission angle $(\theta, \phi)$ as

$$
\chi=\frac{I(\theta, \phi)-I_{0}(\theta)}{I_{0}(\theta)},
$$

where $I_{0}(\theta)$ is the average intensity for each azimuthal scan at polar angle $\theta$. The structural determination was performed by comparing measured XPD patterns to multiple scattering simulations for a trial structure. Such patterns were simulated using the program package for electron diffraction in atomic clusters (EDAC) [32]. The presence of two mirror domain orientations was taken into account by an incoherent superposition of the calculated intensities, based on the well-funded $[33,34]$ assumption of domain sizes that are sufficiently large to neglect boundary effects. The agreement between the simulations and the experimental results was quantified by computing the reliability factor $(R)$ [35],

$$
R=\frac{\sum_{i}\left(\chi_{\exp , i}-\chi_{\operatorname{sim}, i}\right)^{2}}{\sum_{i}\left(\chi_{\exp , i}^{2}+\chi_{\operatorname{sim}, i}^{2}\right)},
$$

where $\chi_{\operatorname{sim}, i}$ and $\chi_{\exp , i}$ are the simulated and the experimental modulation functions for each emission angle $i$. The estimation of the accuracy of the quantities derived by means of $R$-factor analysis, i.e., lattice constant, layer thickness, and percentage of mirror domains, was deduced from the $R$-factor confidence interval defined as [36]

$$
\Delta R_{\min }=R_{\min } \sqrt{\frac{2}{N}},
$$

where $R_{\min }$ is the minimum $R$-factor value and $N$ is the number of well-resolved peaks in the XPD pattern $(N \sim 350)$, i.e., the approximate number of peaks considering the whole 50 azimuthal scans acquired at different polar emission angles. The LEED experiments were carried out using a commercial VG instrument installed at the experimental chamber of the SuperESCA beamline.

LEEM data were recorded using an ELMITEC LEEM III instrument at the University of Bremen. A sample was prepared at the SuperESCA beamline, transferred to Bremen through air, and then annealed to $670 \mathrm{~K}$ after inserting it in the ultrahigh-vacuum (UHV) chamber. As a diffraction-based method, LEEM can access either real space (imaging mode) or reciprocal space (diffraction mode) providing information on both surface morphology and atomic structure [37]. By constricting the illuminated area with an aperture in diffraction mode, information on the local atomic structure can be obtained from areas as small as $250 \mathrm{~nm}$ in diameter, an approach known as $\mu$ LEED. In bright field (BF), imaging contrast usually arises from differences in local atomic structure and composition. In dark field (DF), LEEM diffraction contrast is obtained since only regions contributing to a specific beam will show up bright in the image. The $I(V)$ curves were extracted from a stack of LEEM images, yielding individual $I(V)$ curves for every image pixel, thus enabling structural identification on the nanoscale [38,39].

\section{RESULTS AND DISCUSSION}

The samples were first characterized after the preparation by means of high-resolution XPS. A W $4 f$ spectrum is displayed in Fig. 1(a) and it shows a single peak at binding energy $\mathrm{BE}=32.78 \mathrm{eV}$, characteristic of SL $\mathrm{WS}_{2}$ [34], with its spin-orbit doublet separated by $2.14 \mathrm{eV}$. These spin-orbit split components are not accompanied by contributions at lower binding energy, indicating the absence of incompletely sulfided $\mathrm{W}$ species of the form $\mathrm{WS}_{2-x}(0<x<1)$ or metallic W clusters $[34,40,41]$. The line-shape parameters used for the spectral deconvolution are reported in [31]. 


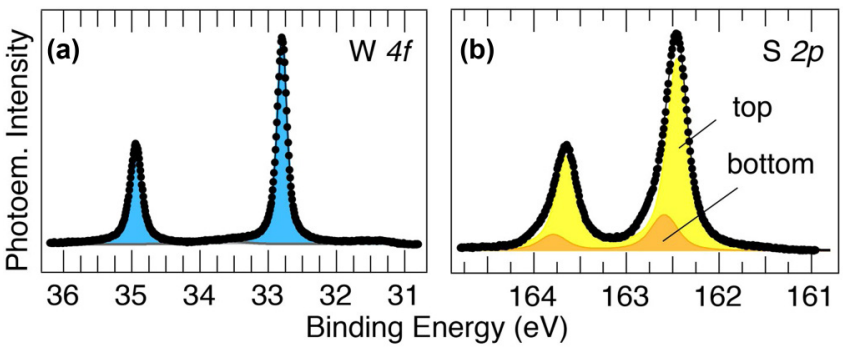

(c)
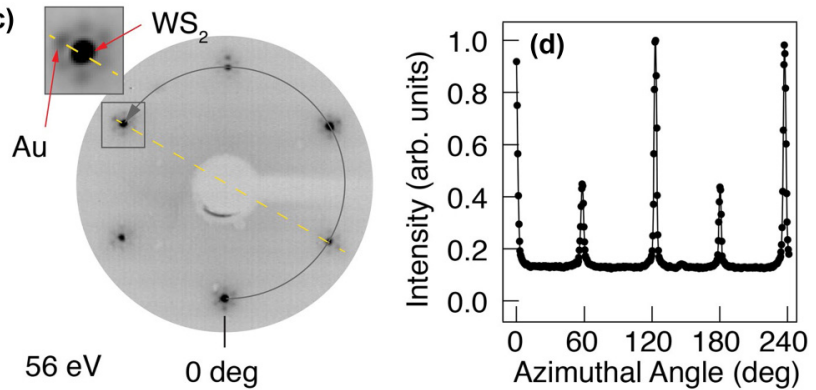

FIG. 1. (a),(b) High-resolution core-level spectra for W $4 f$ $(h v=140 \mathrm{eV})$ and $\mathrm{S} 2 p(h v=260 \mathrm{eV})$, respectively, measured at room temperature (RT). The result of a peak-fitting analysis is displayed as well. (c) LEED pattern (primary electron energy $E_{p}=$ $56 \mathrm{eV}$ ). The dashed yellow line indicates the alignment of the moiré superstructure with the crystallographic orientation of $\mathrm{WS}_{2}$. The solid gray line is a circular profile taken across the $\mathrm{WS}_{2}$ spots and shown in panel (d).

In Fig. 1(b) we report the S $2 p$ spectrum from the same sample. Two main components are visible in each of the spinorbit peaks, indicated in light $\left(\mathrm{S} 2 p_{3 / 2} \mathrm{BE}=162.45 \mathrm{eV}\right)$ and dark $\left(\mathrm{S} 2 p_{3 / 2} \mathrm{BE}=162.59 \mathrm{eV}\right)$ orange: the latter has been assigned to S atoms from the "bottom" [close to the $\mathrm{Au}(111)$ surface] while the former stems from the "top" atoms in the S-W-S sandwich arrangement of $\mathrm{WS}_{2}$. This interpretation follows the assignments for SL $\mathrm{MoS}_{2}$ prepared on $\mathrm{Au}(111)$ [24] and it is confirmed by the XPD experiments aiming to determine the polytype of the SL (see [31]). Similarly to what was observed for the $\mathrm{W} 4 f$ core level, the acquired spectrum for S $2 p$ only shows the spectral components associated with $\mathrm{WS}_{2}$, indicating a better quality of the grown layer.

Figure 1(c) reports the LEED pattern acquired on the sample. The most intense spots corresponding to the reciprocal lattice of $\mathrm{WS}_{2}$ are surrounded by moiré satellites, due to the lattice mismatch between $\mathrm{WS}_{2}$ and the $\mathrm{Au}$ substrate [34]. The first-order $\mathrm{Au}(111)$ diffraction spots also exhibit a hexagonal pattern, though slightly bigger in dimension due to a smaller lattice vector, and they are aligned with those of $\mathrm{WS}_{2}$ [see the dashed yellow line in Fig. 1(e) and the inset]. By comparing the reciprocal lattice vectors of $\mathrm{WS}_{2}$ with those of $\mathrm{Au}(111)$, we obtain a moiré periodicity of $3.19 \pm 0.1 \mathrm{~nm}$, in close agreement with the literature [34]. This is indicative of the formation of a superstructure with a periodicity of $(10 \times 10)-\mathrm{WS}_{2}$ on $(11 \times 11)$-Au unit cell configuration.

Furthermore, the LEED pattern reveals a threefold symmetry in the intensity of the spots (as evidenced by the circular profile taken across the $\mathrm{WS}_{2}$ spots in the pattern), which could indicate the presence of a single azimuthal orientation of the $\mathrm{WS}_{2}$ crystalline domains, given the threefold symmetric structure of the layer. However, a conclusive answer about the orientation of the layers and about the absence of mirror domains can only be obtained by means of XPD experiments.

In XPD, photoemission intensity modulations arise from the interference between the component of the photoelectron wave field that reaches the detector directly from the emitting atom and the components scattered by atoms surrounding the emitter. This makes XPD very sensitive to the local environment of the emitter [35], hence making this technique ideal to address questions about the polytype of the layer (trigonal prismatic or octahedral), the distribution of mirror domains, and other structural parameters such as lattice constant and layer thickness.

We acquired XPD patterns from the $\mathrm{W} 4 f_{7 / 2}$ core level on the layer using a photon energy $h v=170 \mathrm{eV}$ (photoelectron kinetic energy, KE $~ 137 \mathrm{eV}$ ), at which both forward and backward scattering contributions are significant, and at $h v=$ $360 \mathrm{eV}(\mathrm{KE} \sim 326 \mathrm{eV})$, enhancing the forward scattering and suppressing the backscattering [35]. The experimental XPD patterns [color sector in Figs. 2(a) and 2(b)] were compared to simulations (gray scale) using the reliability factor ( $R$-factor) analysis (see Sec. II). Simulated diffraction patterns were calculated for different structural phases and their corresponding structural parameters assuming, as initial guess, the trigonal prismatic $(1 \mathrm{H})$ phase (sketched in Fig. 2), following the indications obtained earlier for this system [34]. The presence of two orientations in the SL can be modeled by assuming an incoherent superposition from the domains, such that the total photoemission intensity is

$$
I_{\mathrm{tot}}=a I_{0}+b I_{\mathrm{mir}} \quad(b=1-a),
$$

where $I_{0}$ is the contribution to the XPD pattern from the main orientation (Or1) and $I_{\text {mir }}$ is the contribution from the mirror orientation (Or2), according to the models shown in the top right of Fig. 2. The simulated XPD patterns for the two mirrored orientations stemming from $\mathrm{W} 4 f_{7 / 2}(\mathrm{KE} \sim 326 \mathrm{eV})$ are reported in Ref. [31].

The threefold symmetry of the simulated patterns for a single orientation matches the experimental data, which is a direct experimental indication of a dominant domain orientation and hints at a minor contribution from the mirror orientation. However, a conclusive determination of the layer orientation was achieved only through the $R$-factor analysis, as shown in the following. For the simulations of the photoemission intensity by multiple scattering calculations, the values for layer thickness (i.e., the vertical S-S distance), the lattice constant, and the fraction of mirror domains in the SL, assuming the $1 \mathrm{H}$ phase, were changed independently, running an $R$-factor optimization in the three-dimensional parameter space, using the data sets of both Figs. 2(a) and 2(b). The analysis clearly reveals a global minimum for a single orientation structure (a maximum of 5\% contribution of mirror domains), a lattice parameter of $3.17 \pm 0.04 \AA$, and a layer thickness of $3.17 \pm 0.05 \AA$, in very good agreement with the values reported in the literature [42]. The very low absolute value of the minimum $R$-factor $(R=0.05)$ indicates an excellent agreement between experiment and simulation. For the sake of simplicity, Fig. 2(c) reports a two-dimensional plot of the $R$-factor as a function of lattice constant and layer thickness obtained for the single orientation $(b=0)$. Figures $2(d)$ and 




FIG. 2. Top panel: Side view (left) of the trigonal prismatic $(1 \mathrm{H})$ structural phase of $\mathrm{WS}_{2}$, and top view (right) of the main (Or1) and mirror (Or2) orientations used for XPD simulations. (a) XPD pattern acquired on the sample for the $\mathrm{W} 4 f_{7 / 2}$ component $(h \nu=170 \mathrm{eV}$ ) (in color) together with a simulation assuming a single orientation of the layer (in gray scale). (b) $\mathrm{W} 4 f_{7 / 2} \mathrm{XPD}$ pattern obtained with $h v=360 \mathrm{eV}$. (c) Contour plot reporting the $R$-factor as a function of the layer thickness and lattice constant. (d) and (e) $R$-factor along the dashed lines in panel (c). (f) $R$-factor vs percentage of mirror orientation admixture, obtained for layer thickness and lattice constant values corresponding to the minima of the $R$-factor shown in panels (d) and (e). The inset of (d), (e). and (f) shows a magnification of the graph around the minimum of the $R$-factor, with the vertical dashed arrow indicating the confidence interval $\Delta R_{\min }$ and the horizontal arrow the uncertainty in the determination of the lattice constant, layer thickness, and percentage of mirror orientation, respectively, as explained in Sec. II. The simulated patterns shown are those obtained employing the parameters deduced from the $R$-factor analysis.

2(e) are obtained by taking cuts along the dashed lines in panel (c) to highlight the $R$-factor trend around the absolute minimum, while Fig. 2(f) clearly shows that a minimum of the $R$-factor is observed when only a single orientation is present. This is considerably different from earlier reports on the growth of $\mathrm{SL} \mathrm{WS}_{2}$ by bottom-up approaches [41], in which the presence of mirror domains could not be avoided.

A similar analysis on the XPD patterns from $S 2 p_{3 / 2}$ and $\mathrm{W} 4 f_{7 / 2}$ was performed assuming an octahedral (1T) polytype for the $\mathrm{WS}_{2} \mathrm{SL}$. The simulations of the XPD patterns were performed using the same lattice constant and layer thickness determined earlier for the $1 \mathrm{H}$ polytype. The analysis returned higher values of the $R$-factor for the $1 \mathrm{~T}$ phase, thus ruling out this structure. The details of this analysis are reported in Ref. [31].

It is interesting to ask which physical mechanisms are responsible for the growth of the single orientation. The only element breaking the symmetry is the $\mathrm{Au}(111)$ substrate.
While the first Au layer has a sixfold symmetry, considering deeper layers lowers the symmetry to threefold, providing a suitable template for the growth of singly oriented $\mathrm{WS}_{2}$. This should then be the main reason behind the single orientation growth. However, the Au surface reconstruction, which is lifted during $\mathrm{WS}_{2}$ growth, could also play a role. Theoretical calculations would be needed in order to shed some light on this issue. In any case, the optimization of the $\mathrm{W}$ deposition rate, substrate temperature, and $\mathrm{H}_{2} \mathrm{~S}$ partial pressure not only improves the quality of the resulting $\mathrm{SL} \mathrm{WS}_{2}$ but drives the formation of a single domain orientation. This could be favorable to achieve because a higher temperature promotes the surface diffusion of $\mathrm{W}$ atoms on the Au substrate, while a lower $\mathrm{W}$ deposition rate, together with a high $\mathrm{H}_{2} \mathrm{~S}$ background pressure, avoids the formation of partially sulfided species, as seen by XPS. These latter species could be the nucleation centers where the domains grow with an orientation that is not the most favorable one. 

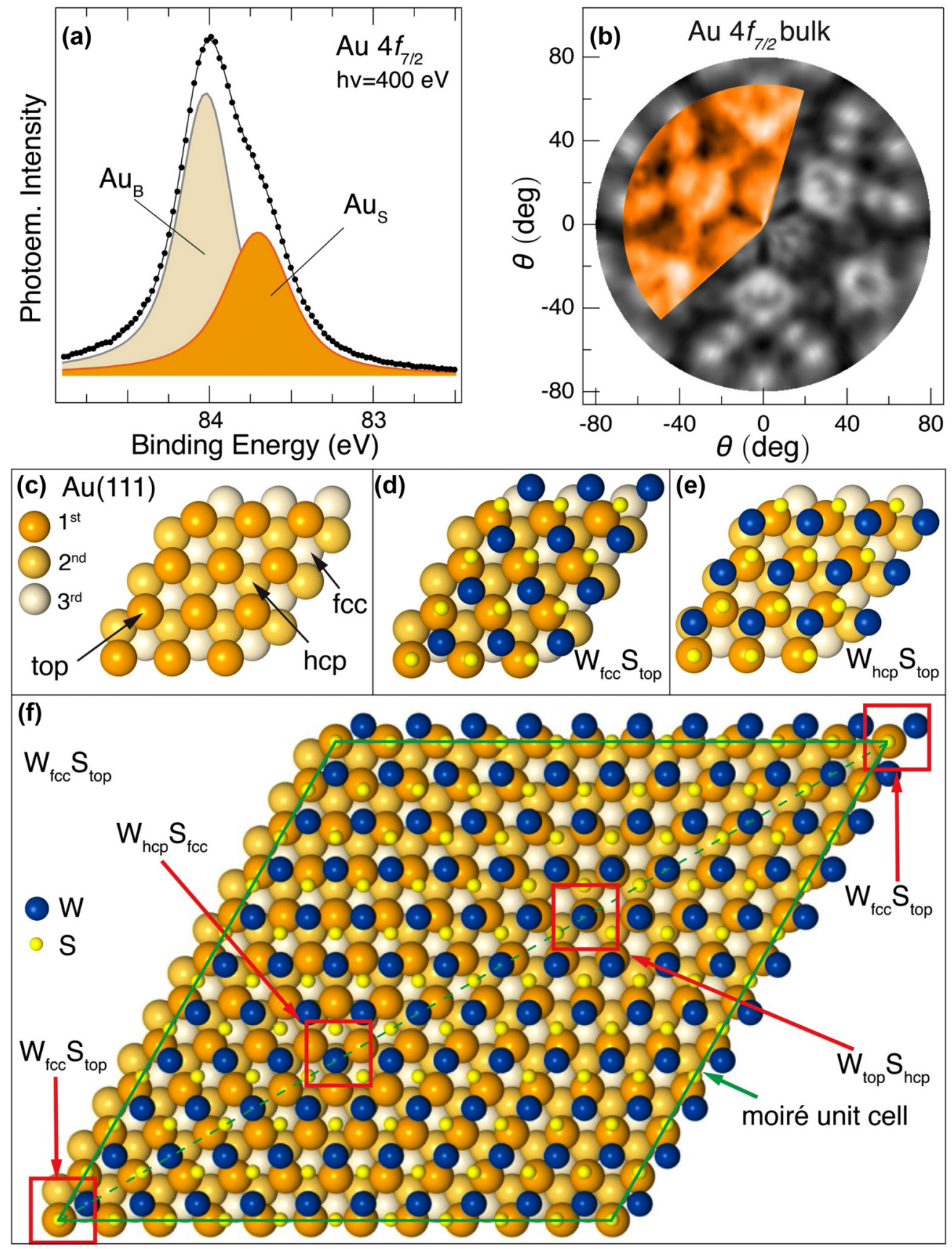

FIG. 3. (a) $\mathrm{Au} 4 f_{7 / 2}$ core level acquired on clean $\mathrm{Au}(111)$ at $h v=400 \mathrm{eV}$. Surface $\left(\mathrm{Au}_{\mathrm{S}}\right)$ and bulk $\left(\mathrm{Au} \mathrm{B}_{\mathrm{B}}\right)$ components are indicated. (b) XPD pattern acquired at $400 \mathrm{eV}$ photon energy $(\mathrm{KE} \sim 316 \mathrm{eV})$, showing the XPD pattern (color) associated with the bulk component $\mathrm{Au}{ }_{B}$ in the $\mathrm{Au} 4 f_{7 / 2}$ core level together with the multiple scattering simulation (gray) for the clean $\mathrm{Au}(111)$ sample. (b) Orientation of the Au sample derived from the comparison of the XPD experiment with the simulation. (d) and (e) Ball model of the adsorption geometry of WS $\mathrm{S}_{2}$ on Au(111) at the corner of the moiré unit cell with orientation Or1 $\left(\mathrm{W}_{\mathrm{fcc}} \mathrm{S}_{\mathrm{top}}\right)$ and Or2 ( $\left.\mathrm{W}_{\mathrm{hcp}} \mathrm{S}_{\mathrm{top}}\right)$, respectively. (f) Moiré unit cell for the configuration Or1 (preferred) with the regions of high local symmetry for $\mathrm{WS}_{2}$ on $\mathrm{Au}(111)$.

Having established that the $\mathrm{WS}_{2}$ layer has a single crystalline orientation, we used the XPD pattern from the $\mathrm{W} 4 f_{7 / 2}$ core-level component to determine the orientation of the $\mathrm{WS}_{2}$ layer with respect to the $\mathrm{Au}(111)$ substrate. To do this, we first determined the orientation of the $\mathrm{Au}(111)$ substrate by measuring an XPD pattern stemming from the bulk component of $\mathrm{Au} 4 f_{7 / 2}$ on the clean Au sample, i.e., before the deposition of $\mathrm{WS}_{2}$.
Figure 3(a) shows the typical Au $4 f_{7 / 2}$ spectrum acquired on clean $\mathrm{Au}(111)$. This is part of the series used to determine the XPD pattern of the bulk component $\mathrm{Au}_{B}$, which is shown (colored sector) in Fig. 3(b) together with a simulated XPD pattern (gray sector) obtained using a four-layer slab, bulkterminated $\mathrm{Au}(111)$ surface, with emitters in the second and deeper layers. A good agreement of the threefold-symmetric pattern [corresponding to the fcc (111) crystal stacking] with 
the simulation $(R$-factor $=0.2)$ returns the orientation of the Au crystal as given in Fig. 3(c). Details about this $R$-factor analysis are given in [31]. Figures 3(d) and 3(e) show the two possible orientations of the $\mathrm{SL} \mathrm{WS}_{2}$ on $\mathrm{Au}(111)$. We call these configurations $\mathrm{W}_{\text {fcc }} S_{\text {top }}$ and $\mathrm{W}_{\text {hcp }} S_{\text {top }}$, in analogy with the case of h-BN on $\operatorname{Ir}(111)$ [26]. In the former configuration, the sulfur atoms at the left bottom corner are adsorbed in the atop position and the $\mathrm{W}$ atoms adsorb in the fcc hollow sites, while in the latter $\mathrm{W}$ sits in hcp hollow sites. The comparison between the XPD patterns of the Au substrate and of the $\mathrm{WS}_{2}$ overlayer unambiguously proves that the singly oriented $\mathrm{WS}_{2}$ sample assumes the configuration $\mathrm{W}_{\mathrm{fcc}} \mathrm{S}_{\text {top }}$ shown in Fig. 3(d). In Fig. 3(e) we show a sketch of the entire moiré unit cell consisting of the $(10 \times 10) \mathrm{WS}_{2}$ superstructure on the $(11 \times 11) \mathrm{Au}$ unit cell for the $\mathrm{W}_{\mathrm{fcc}} \mathrm{S}_{\text {top }}$ orientation.

Additional details about the surface morphology and the size of the crystalline domains have been obtained from LEEM measurements. Figure 4(a) displays a BF image acquired on the sample. A clear contrast is observed on the surface, distinguishing bright and dark areas. Micro-LEED patterns recorded from a $1-\mu \mathrm{m}$ large dark area show the diffraction pattern expected for bare Au substrate (see [31]). On the other hand, the brighter areas are $\mathrm{WS}_{2}$-covered regions, showing the characteristic micro-LEED pattern due to the moiré superlattice [Fig. 4(b)]. An investigation conducted on several images acquired in $\mathrm{BF}$ mode revealed that the average size of the crystalline domains is in the range of a few squared microns.

The use of a DF imaging mode measuring the (10) beam [Fig. 4(c)] should allow for contrast between rotational domains of $\mathrm{WS}_{2}$, given the threefold symmetry of the LEED pattern at some energies, as already evidenced in the LEED pattern of Fig. 1. A DF image of the same surface area is presented in Fig. 4(c). The majority of the $\mathrm{WS}_{2}$ domains show up bright at the chosen electron energy of $31 \mathrm{eV}$. Tuning the energy to $40 \mathrm{eV}$ highlights other $\mathrm{WS}_{2}$ islands, as can be seen in Fig. 4(d). We now show that the islands of different contrast correspond to opposite domain orientations. The dependence of the local LEEM intensity versus electron energy [so-called $I(V)$ LEEM [38]] at the points marked in red and black in Figs. 4(c) and 4(d) can be used to determine the local structure [39]. First, the $I(V)$ curves of the (00) LEED beam for the two domains are compared in Fig. 4(e), revealing almost no difference. This finding directly proves that the two $\mathrm{WS}_{2}$ domains have virtually the same atomic structure $[38,39]$. By contrast, the two $I(V)$ curves obtained for the (10) spot, i.e., for the DF-mode image [Fig. 4(f)], are indeed dissimilar for the two different domains. While at $40 \mathrm{eV}$ one $\mathrm{WS}_{2}$ domain shows intense backreflection, at $31 \mathrm{eV}$ the same domain reflects only weakly while the other does so more strongly. $\mu$ LEED patterns acquired in each of the two regions (see [31]) indicate that they originate from domains with opposite azimuthal alignment. Thus, the domain orientation can be spotted at the nanoscale because the (10) and (01) reflections of the two threefold-symmetric domains appear at the same position in reciprocal space, yielding utterly different $I(V)$ curves. From an overall quantitative evaluation of the available DF images, we derive a fraction of less than $5 \%$ of the surface being covered by the minority domains, in agreement with the results of the $R$-factor analysis performed for the XPD experiments.
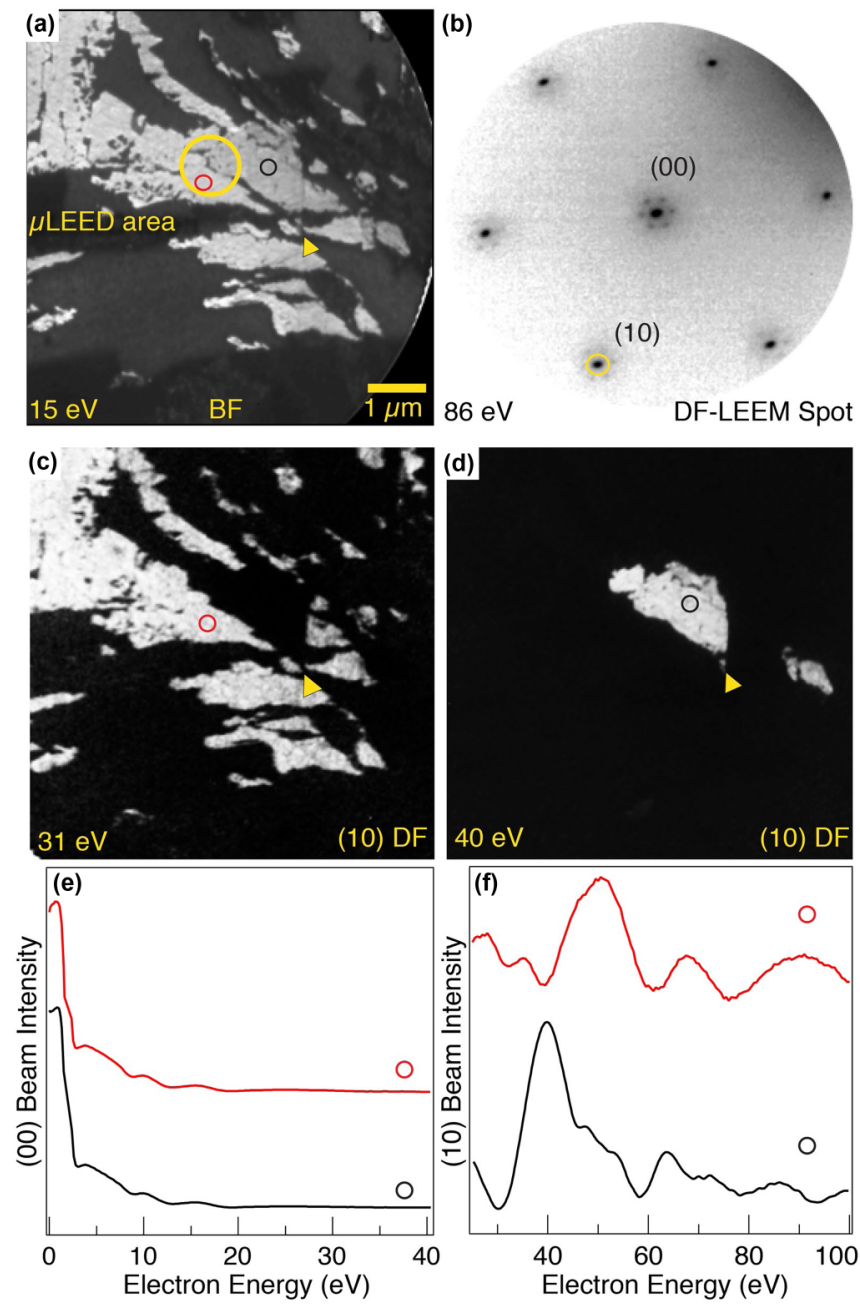

FIG. 4. (a) BF LEEM reveals islands that are identified as $\mathrm{WS}_{2}$ islands by their specific LEED pattern. (b) $\mu$ LEED pattern obtained using an illumination aperture of $1 \mu \mathrm{m}$ [illuminated area marked by a yellow circle in (a)]. (c,d) DF LEEM images of area (a) using the (10) beam of the $\mathrm{WS}_{2}$ LEED pattern [cf. (b)], evidencing contrast changes of different $\mathrm{WS}_{2}$ domains at certain energies. The filled triangle points to a specific reference position in the image. (e) BF $I(V)$ curves from the regions marked with red and black circles. (f) Corresponding DF $I(V)$ curves for the two domains observed in panels (c) and (d), respectively. The $I(V)$ curves are vertically shifted for the sake of clarity.

\section{CONCLUSION}

In summary, we have reported the characterization of the growth and structural features of $\mathrm{WS}_{2}$ single layers on $\mathrm{Au}(111)$. We found that a low $\mathrm{W}$ deposition rate, a high partial pressure of $\mathrm{H}_{2} \mathrm{~S}$, and a high substrate temperature during the growth yield a unique crystalline orientation of $\mathrm{SL} \mathrm{WS}_{2}$, i.e., suppressing the presence of any detrimental mirror domains. The grown layer showed the $1 \mathrm{H}$ polytype (semiconducting), with a lattice parameter of $3.17 \AA$. Moreover, we have observed the formation of a moiré pattern stemming from the overlapping of a $(10 \times 10) \mathrm{WS}_{2}$ layer on a $(11 \times 11)$ Au surface unit cell, arranged with a top-fcc-type stacking. By means of LEEM measurements, we found that 
crystalline domains have an average size of a few squared microns, and we confirmed the presence of a single orientation. Earlier studies about the growth of epitaxial SL $\mathrm{WS}_{2}$ did rarely explore in quantitative detail the domain morphology and orientation. However, there have been indications of a domain mixture different from $50 \%$ for $\mathrm{WS}_{2}$ on $\mathrm{Ag}(111)$, as circular dichroism in the excitation of a valley polarization was observed for this system [43]. The single orientation and high quality of the $\mathrm{WS}_{2}$ samples grown according to the method we have presented make them an ideal candidate for studies about the fundamental, electronic properties of this material. We envision that the layers that we have grown and characterized in this work, combined with methods already available to transfer $\mathrm{WS}_{2}$ from $\mathrm{Au}$ to other substrates [44], can be successfully employed in valleytronics and spintronics devices and applications.

\section{ACKNOWLEDGMENTS}

This work was supported by the Danish Council for Independent Research, Natural Sciences under the Sapere Aude program (Grant No. DFF-4002-00029) and by VILLUM FONDEN via the Centre of Excellence for Dirac Materials (Grant No. 11744).
[1] K. S. Novoselov, A. K. Geim, S. V. Morozov, D. Jiang, Y. Zhang, S. V. Dubonos, I. V. Grigorieva, and A. A. Firsov, Electric field effect in atomically thin carbon films, Science 306, 666 (2004).

[2] K. S. Novoselov, A. K. Geim, S. V. Morozov, D. Jiang, M. I. Katsnelson, I. V. Grigorieva, S. V. Dubonos, and A. A. Firsov, Two-dimensional gas of massless Dirac fermions in graphene, Nature (London) 438, 197 (2005).

[3] Y. B. Zhang, Y. W. Tan, H. L. Stormer, and P. Kim, Experimental observation of the quantum Hall effect and Berry's phase in graphene, Nature (London) 438, 201 (2005).

[4] K. S. Novoselov, D. Jiang, F. Schedin, T. J. Booth, V. V. Khotkevich, S. V. Morozov, and A. K. Geim, Two-dimensional atomic crystals, Proc. Natl. Acad. Sci. (U.S.A.) 102, 10451 (2005)

[5] A. Splendiani, L. Sun, Y. Zhang, T. Li, J. Kim, C.-Y. Chim, G. Galli, and F. Wang, Emerging photoluminescence in monolayer $\mathrm{MoS}_{2}$, Nano Lett. 10, 1271 (2010).

[6] K. F. Mak, C. Lee, J. Hone, J. Shan, and T. F. Heinz, Atomically Thin $\mathrm{MoS}_{2}$ : A New Direct-Gap Semiconductor, Phys. Rev. Lett. 105, 136805 (2010).

[7] B. Radisavljevic, A. Radenovic, J. Brivio, V. Giacometti, and A. Kis, Single-layer $\mathrm{MoS}_{2}$ transistors, Nat. Nanotechnol. 6, 147 (2011).

[8] O. Lopez-Sanchez, D. Lembke, M. Kayci, A. Radenovic, and A. Kis, Ultrasensitive photodetectors based on monolayer $\mathrm{MoS}_{2}$, Nat. Nanotechnol. 8, 497 (2013).

[9] D. Xiao, G.-B. Liu, W. Feng, X. Xu, and W. Yao, Coupled Spin and Valley Physics in Monolayers of $\mathrm{MoS}_{2}$ and Other Group-VI Dichalcogenides, Phys. Rev. Lett. 108, 196802 (2012).

[10] K. F. Mak, K. He, J. Shan, and T. F. Heinz, Control of valley polarization in monolayer $\mathrm{MoS}_{2}$ by optical helicity, Nat. Nanotechnol. 7, 494 (2012).

[11] H. Zeng, J. Dai, W. Yao, D. Xiao, and X. Cui, Valley polarization in $\mathrm{MoS}_{2}$ monolayers by optical pumping, Nat. Nanotechnol. 7, 490 (2012).

[12] T. Cao, G. Wang, W. Han, H. Ye, C. Zhu, J. Shi, Q. Niu, P. Tan, E. Wang, B. Liu, and J. Feng, Valley-selective circular dichroism of monolayer molybdenum disulphide, Nat. Commun. 3, 887 (2012).

[13] K. F. Mak, K. L. McGill, J. Park, and P. L. McEuen, The valley Hall effect in $\mathrm{MoS}_{2}$ transistors, Science 344, 1489 (2014).

[14] X. Xu, W. Yao, D. Xiao, and T. F. Heinz, Spin and pseudospins in layered transition metal dichalcogenides, Nat. Phys. 10, 343 (2014).
[15] Y.-H. Lee, X.-Q. Zhang, W. Zhang, M.-T. Chang, C.-T. Lin, K.-D. Chang, Y.-C. Yu, J. T.-W. Wang, C.-S. Chang, L.-J. $\mathrm{Li}$, and T.-W. Lin, Synthesis of large-area $\mathrm{MoS}_{2}$ atomic layers with chemical vapor deposition, Adv. Mater. 24, 2320 (2012).

[16] Y. Shi, W. Zhou, A.-Y. Lu, W. Fang, Y.-H. Lee, A. L. Hsu, S. M. Kim, K. K. Kim, H. Y. Yang, L.-J. Li, J.-C. Idrobo, and J. Kong, van der Waals epitaxy of $\mathrm{MoS}_{2}$ layers using graphene as growth templates, Nano Lett. 12, 2784 (2012).

[17] M.-Y. Li, Y. Shi, C.-C. Cheng, L.-S. Lu, Y.-C. Lin, H.-L. Tang, M.-L. Tsai, C.-W. Chu, K.-H. Wei, J.-H. He, W.-H. Chang, K. Suenaga, and L.-J. Li, Epitaxial growth of a monolayer $\mathrm{WSe}_{2}-\mathrm{MoS}_{2}$ lateral p-n junction with an atomically sharp interface, Science 349, 524 (2015).

[18] J. J. Pyeon, S. H. Kim, D. S. Jeong, S.-H. Baek, C.-Y. Kang, J.-S. Kim, and S. K. Kim, Wafer-scale growth of $\mathrm{MoS}_{2}$ thin films by atomic layer deposition, Nanoscale 8, 10792 (2016).

[19] O. Lehtinen, H.-P. Komsa, A. Pulkin, M. B. Whitwick, M.-W. Chen, T. Lehnert, M. J. Mohn, O. V. Yazyev, A. Kis, U. Kaiser, and A. V. Krasheninnikov, Atomic scale microstructure and properties of Se-deficient two-dimensional $\mathrm{MoSe}_{2}$, ACS Nano 9, 3274 (2015).

[20] X. Chen, Y. J. Park, T. Das, H. Jang, J.-B. Lee, and J.-H. Ahn, Lithography-free plasma-induced patterned growth of $\mathrm{MoS}_{2}$ and its heterojunction with graphene, Nanoscale 8, 15181 (2016).

[21] D. Fu, X. Zhao, Y.-Y. Zhang, L. Li, H. Xu, A.-R. Jang, S. I. Yoon, P. Song, S. M. Poh, T. Ren, Z. Ding, W. Fu, T. J. Shin, H. S. Shin, S. T. Pantelides, W. Zhou, and K. P. Loh, Molecular beam epitaxy of highly crystalline monolayer molybdenum disulfide on hexagonal boron nitride, J. Am. Chem. Soc. 139 9392 (2017).

[22] W. T. Kang, I. M. Lee, S. J. Yun, Y. Il Song, K. Kim, D.-H Kim, Y. S. Shin, K. Lee, J. Heo, Y.-M. Kim, Y. H. Lee, and W. J. Yu, Direct growth of doping controlled monolayer $\mathrm{WSe}_{2}$ by selenium-phosphorus substitution, Nanoscale 10, 11397 (2018).

[23] A. Rycerz, J. Tworzydlo, and C. W. J. Beenakker, Valley filter and valley valve in graphene, Nat. Phys. 3, 172 (2007).

[24] H. Bana, E. Travaglia, L. Bignardi, P. Lacovig, C. E Sanders, M. Dendzik, M. Michiardi, M. Bianchi, D. Lizzit, F. Presel, D. D. Angelis, N. Apostol, P. K. Das, J. Fujii, I. Vobornik, R. Larciprete, A. Baraldi, P. Hofmann, and S. Lizzit, Epitaxial growth of single-orientation high-quality $\mathrm{MoS}_{2}$ monolayers, $2 \mathrm{D}$ Mater. 5, 035012 (2018). 
[25] K. F. Mak and J. Shan, Photonics and optoelectronics of 2D semiconductor transition metal dichalcogenides, Nat. Photon. 10, 216 (2016).

[26] F. Orlando, P. Lacovig, L. Omiciuolo, N. G. Apostol, R. Larciprete, A. Baraldi, and S. Lizzit, Epitaxial growth of a single-domain hexagonal boron nitride monolayer, ACS Nano 8, 12063 (2014).

[27] Z. Y. Zhu, Y. C. Cheng, and U. Schwingenschlögl, Giant spin-orbit-induced spin splitting in two-dimensional transitionmetal dichalcogenide semiconductors, Phys. Rev. B 84, 153402 (2011).

[28] Q. H. Wang, K. Kalantar-Zadeh, A. Kis, J. N. Coleman, and M. S. Strano, Electronics and optoelectronics of two-dimensional transition metal dichalcogenides, Nat. Nanotechnol. 7, 699 (2012).

[29] A. Kormányos, G. Burkard, M. Gmitra, J. Fabian, V. Zólyomi, N. D. Drummond, and V. Fal'ko, $k \cdot p$ theory for twodimensional transition metal dichalcogenide semiconductors, 2D Mater. 2, 022001 (2015).

[30] A. Baraldi, G. Comelli, S. Lizzit, M. Kiskinova, and G. Paolucci, Real-time x-ray photoelectron spectroscopy of surface reactions, Surf. Sci. Rep. 49, 169 (2003).

[31] See Supplemental Material at http://link.aps.org/supplemental/ 10.1103/PhysRevMaterials.3.014003 for the line shape of the core levels, the characterization of the $\mathrm{WS}_{2}$ growth with fastXPS, the determination of the orientation of the Au substrate, the determination of the $\mathrm{WS}_{2}$ polytype, and additional XPD and LEEM data.

[32] F. J. García de Abajo, M. A. Van Hove, and C. S. Fadley, Multiple scattering of electrons in solids and molecules: A cluster-model approach, Phys. Rev. B 63, 075404 (2001).

[33] S. S. Grønborg, S. Ulstrup, M. Bianchi, M. Dendzik, C. E. Sanders, J. V. Lauritsen, P. Hofmann, and J. A. Miwa, Synthesis of epitaxial single-layer $\mathrm{MoS}_{2}$ on $\mathrm{Au}(111)$, Langmuir 31, 9700 (2015).

[34] M. Dendzik, M. Michiardi, C. Sanders, M. Bianchi, J. A. Miwa, S. S. Grønborg, J. V. Lauritsen, A. Bruix, B. Hammer, and
P. Hofmann, Growth and electronic structure of epitaxial singlelayer $\mathrm{WS}_{2}$ on Au(111), Phys. Rev. B 92, 245442 (2015).

[35] D. P. Woodruff, Adsorbate structure determination using photoelectron diffraction: Methods and applications, Surf. Sci. Rep. 62, 1 (2007).

[36] J. B. Pendry, Reliability factors for LEED calculations, J. Phys. C 13, 937 (1980).

[37] J. I. Flege, W.-X. Tang, and M. S. Altman, Low-energy electron microscopy, in Characterization of Materials, edited by E. N. Kaufmann (Wiley, Hoboken, NJ, 2012), pp. 1808-1827.

[38] J. I. Flege and E. E. Krasovskii, Intensity-voltage low-energy electron microscopy for functional materials characterization, Phys. Status Solidi-Rapid Res. Lett. 8, 463 (2014).

[39] J. I. Flege, B. Herd, J. Goritzka, H. Over, E. E. Krasovskii, and J. Falta, Nanoscale origin of mesoscale roughening: Realtime tracking and identification of three distinct ruthenium oxide phases in ruthenium oxidation, ACS Nano 9, 8468 (2015).

[40] A. P. Shpak, A. M. Korduban, L. M. Kulikov, T. V. Kryshchuk, N. B. Konig, and V. O. Kandyba, XPS studies of the surface of nanocrystalline tungsten disulfide, J. Electron Spectrosc. Relat. Phenom. 181, 234 (2010).

[41] H. G. Fuchtbauer, A. K. Tuxen, P. G. Moses, H. Topsoe, F. Besenbacher, and J. V. Lauritsen, Morphology and atomic-scale structure of single-layer $\mathrm{WS}_{2}$ nanoclusters, Phys. Chem. Chem. Phys. 15, 15971 (2013).

[42] W. J. Schutte, J. L. De Boer, and F. Jellinek, Crystal structures of tungsten disulfide and diselenide, J. Solid State Chem. (France) 70, 207 (1987).

[43] S. Ulstrup, A. Grubišić Čabo, D. Biswas, J. M. Riley, M. Dendzik, C. E. Sanders, M. Bianchi, C. Cacho, D. Matselyukh, R. T. Chapman, E. Springate, P. D. C. King, J. A. Miwa, and P. Hofmann, Spin and valley control of free carriers in single-layer $\mathrm{WS}_{2}$, Phys. Rev. B 95, 041405 (2017).

[44] Y. Gao, Z. Liu, D.-M. Sun, L. Huang, L.-P. Ma, L.-C. Yin, T. Ma, Z. Zhang, X.-L. Ma, L.-M. Peng, H.-M. Cheng, and W. Ren, Large-area synthesis of high-quality and uniform monolayer $\mathrm{WS}_{2}$ on reusable Au foils, Nat. Commun. 6, 8569 (2015). 